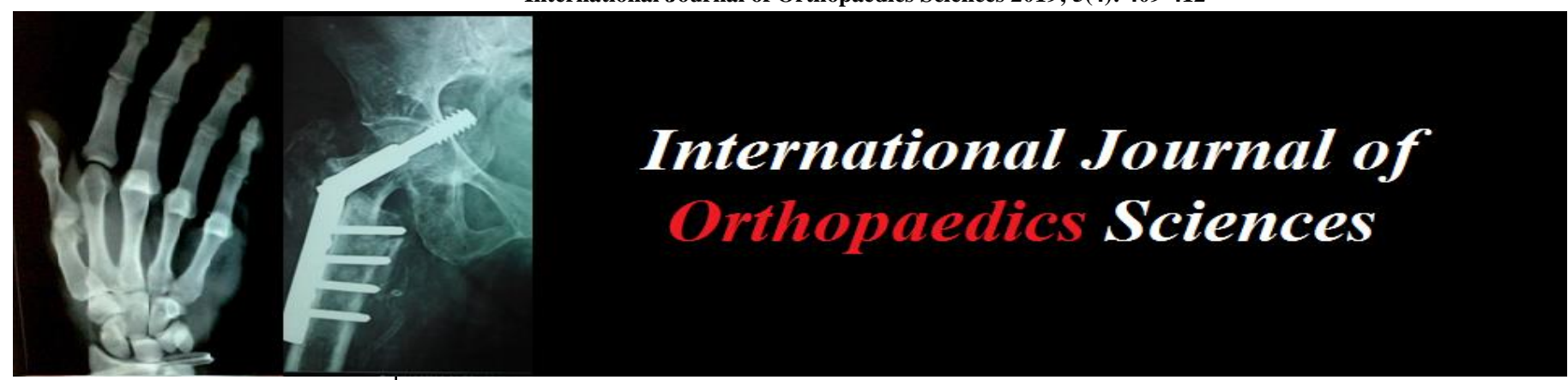

E-ISSN: 2395-1958

P-ISSN: 2706-6630

IJOS 2019; 5(4): 409-412

(C) 2019 IJOS

www.orthopaper.com

Received: 01-08-2019

Accepted: 05-09-2019

Dr. Mithun Shetty

AJ Institute of Medical Scince and Research Center Mangalore, Karnataka, India

Dr. Avinash TP

AJ Institute of Medical Scince and Research Center Mangalore,

Karnataka, India

Dr. Sudarshan Bhandary

AJ Institute of Medical Scince and Research Center Mangalore, Karnataka, India

\section{Split depression fracture of medial tibial plateau, seldom isolated: An interesting insight}

\author{
Dr. Mithun Shetty, Dr. Avinash TP and Dr. Sudarshan Bhandary
}

DOI: https://doi.org/10.22271/ortho.2019.v5.i4h.1707

\section{Abstract}

Significance of isolated split depression fracture of medial tibial plateau is less understood in contrast to lateral tibial plateau fractures. Extreme forces are required to fracture medial tibial plateau which has a thicker and stronger construct of cartilage and subchondral bone, this makes it highly unlikely to occur in isolation. In this case report of isolated split depression fracture of medial tibial plateau, we would like to discuss the possible cause, mechanism of injury and the importance of identifying the concomitant injuries. Which would significantly alter the course of management and functional outcome.

Keywords: Split depression, medial tibial plateau, seldom isolated

\section{Introduction}

Tibial plateau fractures are complex intra-articular fractures which are extremely challenging to treat, owing to their association with numerous debilitating complications ${ }^{[1]}$. Neurovascular injury, impairment of soft tissue, inconspicuous nature of joint and associated ligamentous injury renders the management of these fractures more challenging $[1,2,3]$. Unlike lateral condyle fractures of the tibial plateau, isolated involvement of medial tibial condyle is a rare entity. Moreover, valgus force is a known mechanism of injury for inducing lateral condyle fractures of tibia with or without involvement of ligaments of the opposite corner. In contrast to lateral tibial plateau, medial plateau is larger in surface area and has a thicker and stronger construct of cartilage and subchondral bone, hence requires a high energy trauma to create a medial condyle fracture ${ }^{[4]}$. Such high velocity injuries is more likely to create a bicondylar fracture of proximal tibia. Isolated medial condyle fractures sparing the lateral condyle is a result of unconventional forces and hence it is imperative to be circumspective when we encounter these fractures. Here we discuss a case of split depression fracture of medial tibial plateau sparing the lateral condyle and share an insight over what could be the most probable mechanism of injury after an extensive review of existing literature.

\section{Case Report}

37 year old male presented with history of fall from coconut tree of about $5 \mathrm{ft}$ height and sustained injury to left knee. Patient had pain and swelling of left knee and inability to actively dorsiflex left ankle following the fall. On examination there was tenderness, effusion and painful restriction of movements in the left knee joint. On clinical evaluation it was evident that there was foot drop in the affected limb. Special tests for assessment of ligament injuries was not performed in this acute scenario, although knee appeared grossly unstable. Associated injuries were ruled out after an head to toe examination. Radiographs of left knee confirmed presence of medial tibial plateau fracture and fracture of the fibular head (figure 01). Patient was further evaluated with CT scan to study the intra-articular fracture pattern and MRI was done to assess the ligamentous anatomy. 

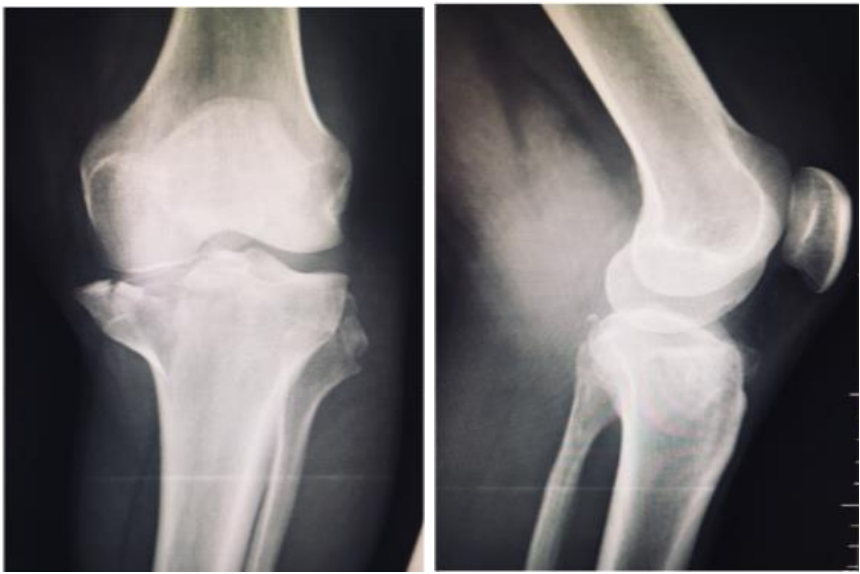

Fig 1: Radiographs demonstrating medial tibial plateau fracture and fracture of the fibular head

CT scan showed split fracture of medial tibial plateau with a depressed fragment in the center of the medial plateau. Avulsion fracture of the upper end of fibula was also noted. Lateral condyle was spared and there were no avulsion fractures of posterior or anterior cruciate ligaments. (fig 02)
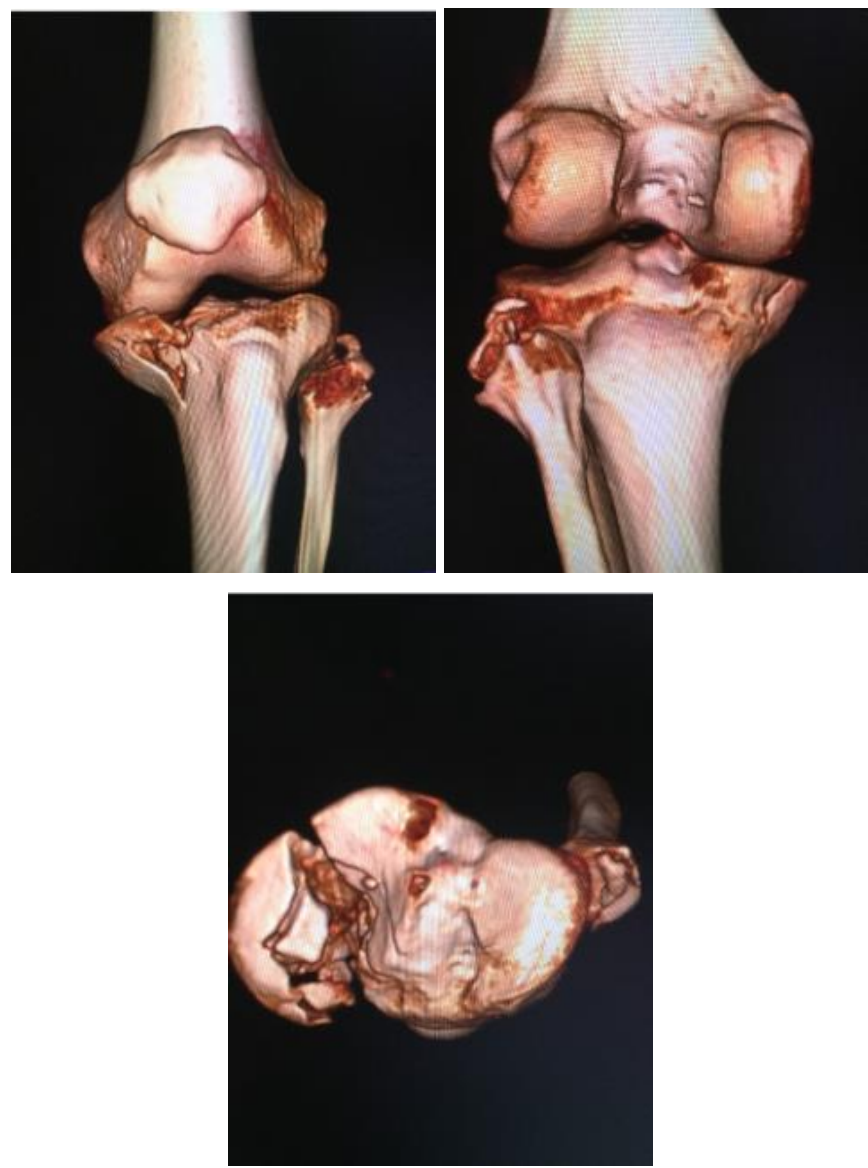

Fig 2: CT 3D Reconstruction image showing split fracture of medial tibial plateau with a depressed fragment in the center and avulsion fracture of the upper end of the fibula

MRI of left knee showed displaced fracture of medial condyle of tibia with intra-articular extension and bone marrow edema. Avulsion fracture of fibular head was present with edema in lateral aspect of the knee joint. Vertical tear of body of medial meniscus noted. There was no clear cut evidence of rupture of any of the knee ligaments although lateral and postero-lateral corner soft tissues of left knee appeared edematous.

\section{Procedure}

Patient was positioned supine in a radiolucent operating table. With the medial approach of the knee, fracture window was opened by incising medial collateral ligament (MCL) in line with fracture. (fig 03). Depressed central fragment was elevated through the fracture window and temporarily fixed with K-wire under fluoroscopy guidance(fig 04) and bone substitute (G BONE -it is mixture of hydroxyapatite and tricalcium phosphatase and other forms of calcium such as calcium carbonate an bi calcium phosphatase) was applied for preventing further collapse. Medial condyle fracture was anatomically reduced and fixed with 3.5 system pre-contoured anatomical plate. Two screws running parallel to the joint was placed through the plate, effectively acting as raft screws.

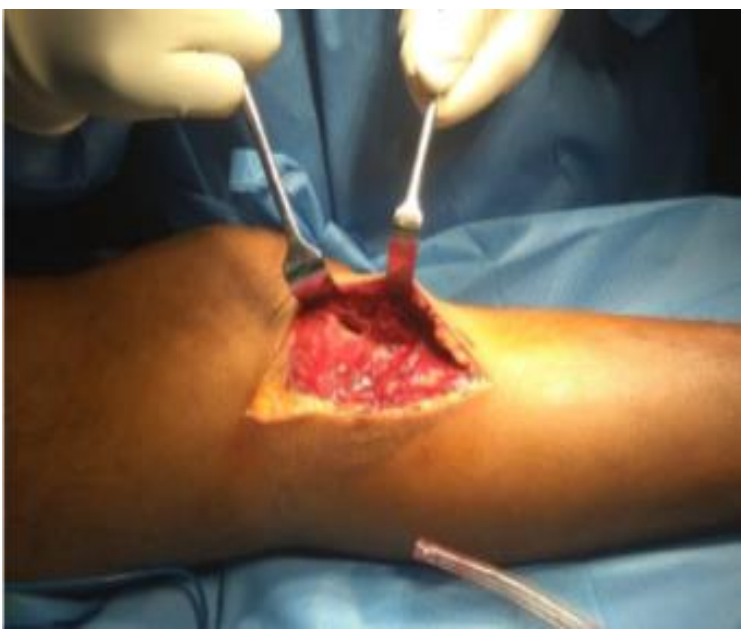

Fig 3: medial approach

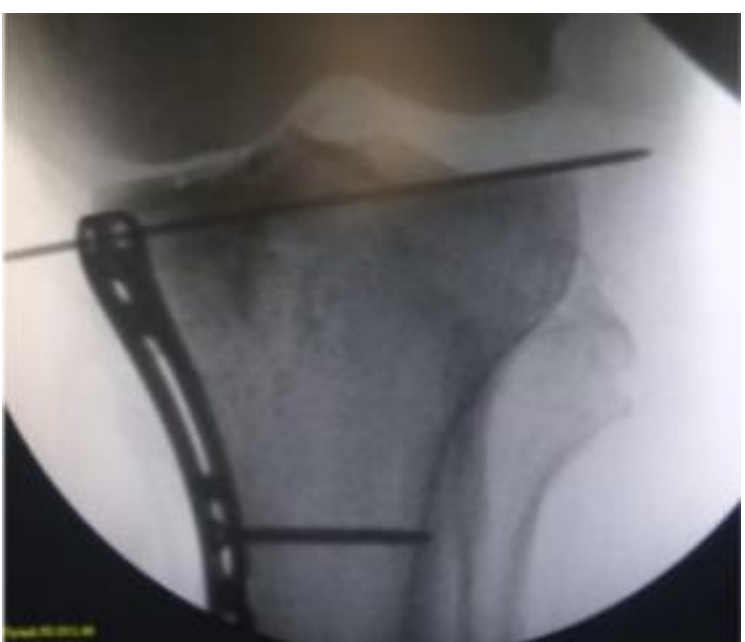

Fig 4: Prefixation with k-wire after reduction)

Lateral opening up of knee joint persisted even after the medial condyle fixation suggesting injury to lateral structures. Lateral side of the knee was approached by an incision running along the biceps tendon upto the fibular head and neck. Common peroneal nerve was identified (fig 05) and explored up to the fibular neck, which was contused and was intact throughout its course in the postero-lateral aspect of knee. Biceps femoris tendon and fibular collateral ligament both were identified and traced up to the avulsed fragment of fibular head. A double loaded 5mm suture anchor was used for fixation of avulsion fracture of fibular head. Random stitches were taken to the torn capsule and soft tissues around the lateral and posterolateral corner of knee which added to the stability of knee. 


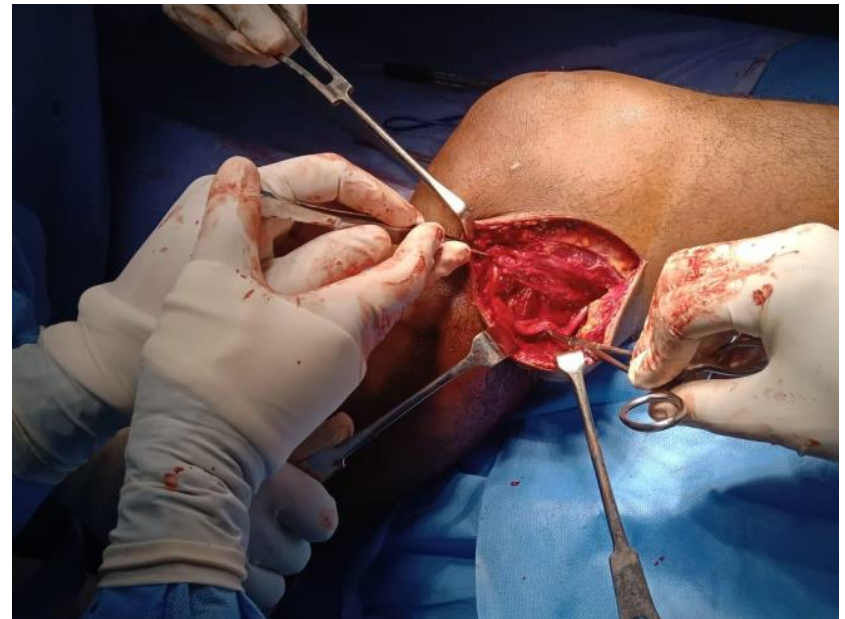

Fig 5: Lateral approach- Isolation of common peroneal nerve

Patient was immobilized in a range of motion brace for 6 weeks (fig 08) and no more than 30 degree range of motion was allowed. Patient had no signs of recovery of common peroneal nerve injury and hence asked to follow up on timely basis for reassessment.
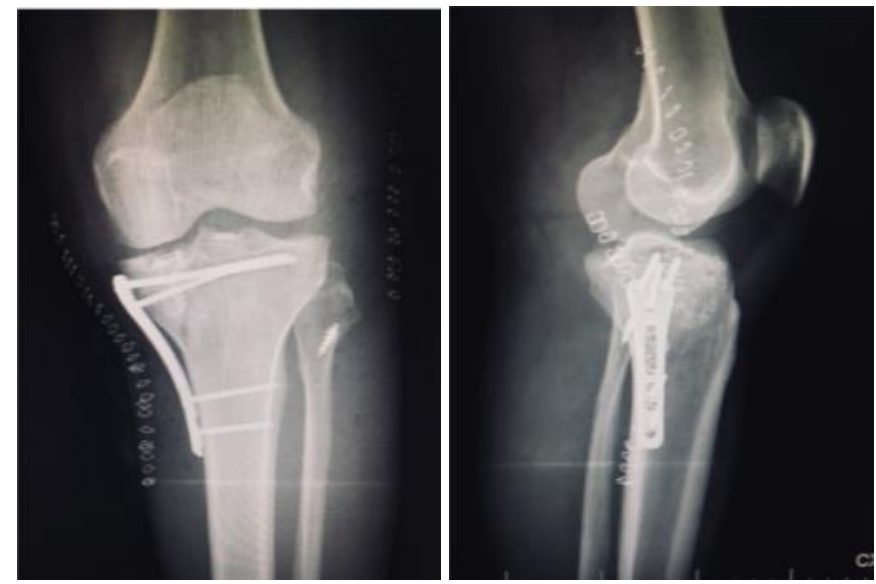

Fig 6: Immediate post-op
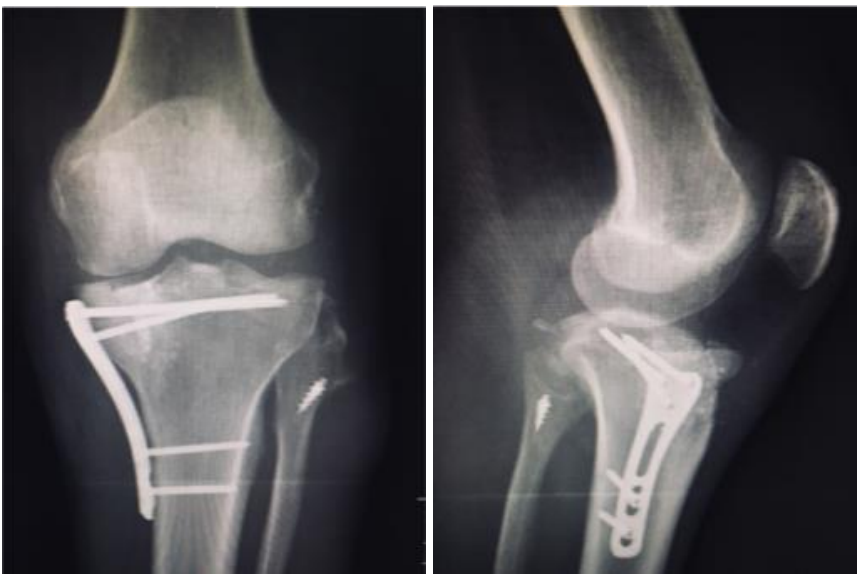

Fig 7-6: weeks post op

\section{Discussion}

Lateral condyle fractures of proximal tibia are relatively more common and has been extensively studied in literature with well proposed mechanism of injury ${ }^{[4]}$. Since it is a relatively a low energy trauma that is responsible for isolated lateral condyle fractures, associated neurological and ligamentous injuries are minimal and most of them do not require active management ${ }^{[5]}$. In contrast to this, isolated medial tibial plateau injuries are less understood ${ }^{[6]}$. Although type IV schatzker injuries and few of its variants have been explained by varus and sheer forces, an in-depthanalysis of complex injuries are not mentioned in literature ${ }^{[4]}$. Distinctly split depression fractures of medial tibial condyle are categorized under section 41.B 3.2 in Arbeitsgemeinschaft fur Osteosynthesefragen (AO) classification system. This fracture pattern do not fit into any of the types described in Schatzker or Hohl classification system precisely. Moreover literature do not provide any explanation on the mechanism of injury of this specific fracture pattern. Shi-Min Chang has made an attempt of providing morphological sub classification using CT scan. However it lacks the explanation precisely on split depression fractures of medial tibial plateau combined with ligamentous injuries. In our case there was a split depression type of medial tibial plateau fracture without any involvement of lateral condyle. Split depression pattern is described frequently in lateral condyle fractures and occasionally can be seen on medial plateau in bicondylar fractures.

Medial condyle fractures in general are considered occult dislocations and hence associated neurovascular and ligamentous injury is anticipatory. Generally there is a conspicuous subluxation of the knee joint in medial condyle fractures with fracture line running into the tibial eminence in the midline ${ }^{[8]}$. Unlike these fractures split depression variety of medial condyle fractures appears more benign without any subluxation. In our case radiographs taken in both the views did not show any evidence of subluxation making it apparently a benign injury. However in this case, the presence of minimally displaced fibular head fracture, with a background of existing foot drop should evoke a suspicion not only on the severity of injury but also on the mechanism of injury. Direct impact on the lateral corner of the knee is responsible for valgus force, which in combination with the sheer force can cause lateral condyle fractures with or without associated opposite corner injuries. However it is unlikely to have a direct impact on medial corner of the knee since the contralateral limb shields any direct forces on to the medial aspect. Hence fall from height would be more likely mechanism of injury to create a varus bending force, due to eccentric loading of the limb while landing. Considering the mechanism of injury, it can be contemplated that medial condyle split depression fracture occurs subsequently as a result of failure of ligamentous structures stabilizing the lateral corner of the knee. It is essential to tackle the lateral structures to prevent instability of the knee and also prudent to explore the common peroneal nerve and visualize the structural integrity of the nerve ${ }^{[10]}$.

Medial compartment of the knee bears more load when compared to the lateral compartment. It has a larger surface area and thicker articular cartilage with stronger subchondral bone ${ }^{[4]}$. Hence it can be concluded that it needs high energy compressive force to create a split depression of the medial tibial plateau. With high energy compressive forces, bicondylar fractures are more likely to occur and hence compressive forces alone causing this fracture pattern is dubious. A combination of compressive force in the medial compartment and distractive force in the lateral compartment can occur due to varus angulation. Hence it can be contemplated that, these fracture patterns have a complex mechanism of injuries ${ }^{[1]}$. Commonly associated injuries with medial tibial condyle fractures are lateral collateral injury, biceps tendon injury, posterolateral corner injuries, meniscal tears, common peroneal nerve injury and posterior cruciate ligament injuries ${ }^{[10]}$. 
Proposed mechanism

A. Axial load + Varus angulation

B. Axial load + Varus angulation + Hyperextension

C. Axial load + Varus angulation + Rotational forces

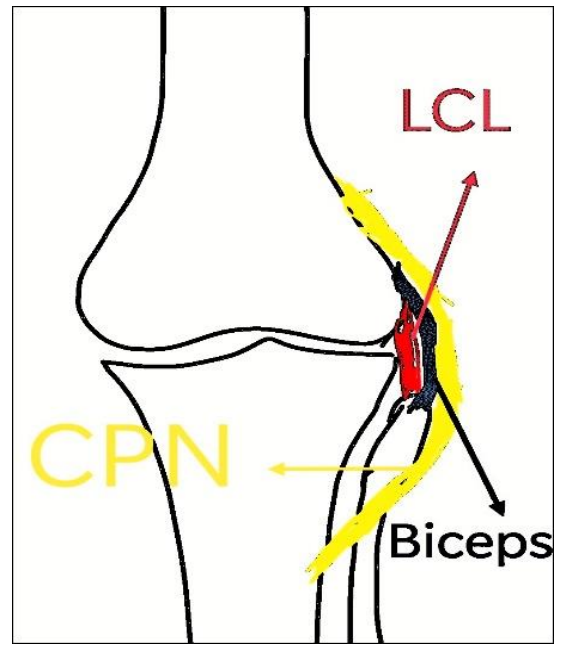

Fig 8: Axial force is mainly responsible for the plateau fractures and meniscal injuries.

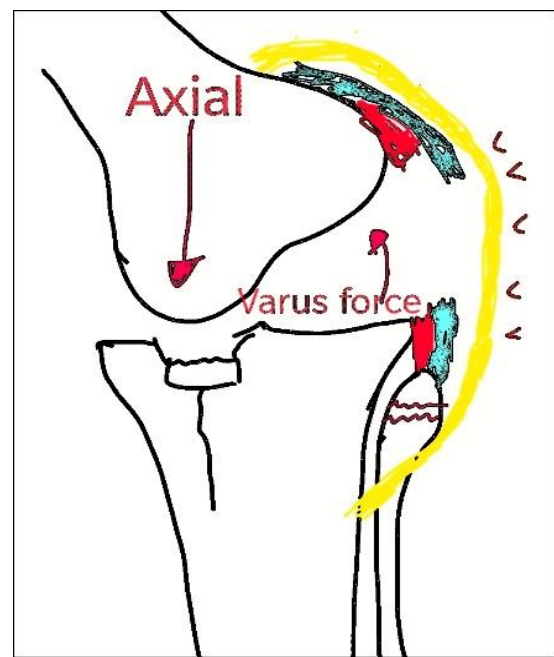

Fig 9: Varus angulation is mainly responsible for the injuries of lateral collateral ligament, biceps tendon, common peroneal nerve injury and avulsion fractures of the fibular head in varying combination

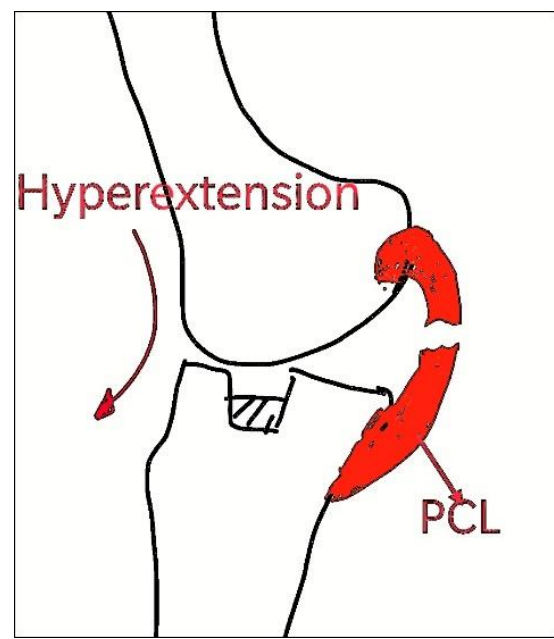

Fig 10: Hyperextension and rotational forces are responsible for posterior cruciate ligament injuries, posterolateral corner injuries and vascular injuries. However, extreme forces can involve any ligamentous and neurovascular structures around the knee.

\section{Conclusion}

Unlike other tibial condyle fractures these fractures appear benign on radiographic examinations but as a rule it is very consistent in having concealed neurovascular and ligamentous injuries. It is imperative to study split depression fractures of medial tibial plateau as a complex injury and never in isolation. These fractures are rare ${ }^{[11]}$ and belong to "AO 41.B.3.2 Complex". Due to their association with injuries of relentless nature which when unnoticed or ignored during the management can have a deteriorating outcome, these fractures could be more appropriately called the "Quagmire fractures".

\section{References}

1. Bennett WF, Browner B. Tibial plateau fractures: a study of associated soft tissue injuries. Journal of orthopaedic trauma. 1994; 8(3):183-8.

2. Delamarter RB, Hohl M, Hopp JE. Ligament injuries associated with tibial plateau fractures. Clinical orthopaedics and related research. 1990; (250):226-33.

3. Sabesan VJ, Danielsky PJ, Childs A, Valikodath T. Multiligament knee injuries with associated tibial plateau fractures: A report of two cases. World journal of orthopedics. 2015; 6(3):363.

4. Kfuri M, Schatzker J. Revisiting the Schatzker classification of tibial plateau fractures. Injury. 2018; 49(12):2252-63.

5. Millán-Billi A, Gómez-Masdeu M, Ramírez-Bermejo E, Ibañez M, Gelber PE. What is the most reproducible classification system to assess tibial plateau fractures? International orthopaedics. 2017; 41(6):1251-6.

6. Gicquel T, Najihi N, Vendeuvre T, Teyssedou S, Gayet LE, Huten D. Tibial plateau fractures: reproducibility of three classifications (Schatzker, AO, Duparc) and a revised Duparc classification. Orthopaedics\& Traumatology: Surgery \& Research. 2013; 99(7):805-16.

7. Chang SM, Li Q, Guo Z, Qi Y, Yao MW, Du SC. Schatzker Type IV Medial Tibial Plateau Fractures: A Computed Tomography-based Morphological Subclassification. Orthopedics. 2014; 37(8):e699-706.

8. Swenson TM. Physical diagnosis of the multipleligament-injured knee. Clinics in sports medicine. 2000; 19(3):415-23.

9. Cush GJ, Maloney PJ, Irgit K. Drop foot after knee dislocation: evaluation and treatment. In The Multiple Ligament Injured Knee. Springer, New York, NY. 2013, pp. 343-353.

10. Recondo JA, Salvador E, Villanúa JA, Barrera MC, Gervás, Alústiza JM. Lateral stabilizing structures of the knee: functional anatomy and injuries assessed with MR imaging. Radiographics. 2000; 20(suppl_1):S91-102.

11. Muhamed Wasil NP, Shetty S, Shetty M. A case of medial tibial plateau split depressed fracture treated with ORIF \& plating with bone substitute. International Journal of Orthopaedics. 2019; 5(2):216-8. 